\title{
Enhancing food engineering education with interactive web-based simulations
}

\author{
Alexandros Koulouris ${ }^{a^{*}}$, Georgios Aroutidis ${ }^{\mathrm{a}}$, Dimitris Vardalis ${ }^{\mathrm{a}}$, Petros \\ GiAnNOUlis $^{\mathrm{a}}$, AND PARASKEVI KARAKOSTA ${ }^{\mathrm{a}}$ \\ ${ }^{a}$ Department of Food Technology, Alexander Technological Education Institute of Thessaloniki, P.O. Box 141, \\ 57400 Thessaloniki, Greece \\ ${ }^{*}$ Corresponding author \\ akoul@food.teithe.gr \\ TEL: +30-2310013576 \\ Received: 10 June 2014; Published online: 18 April 2015 \\ Invited paper from the $3^{\text {rd }}$ International ISEKI_Food Conference - ISEKI_Food 2014 - Bridging Training and \\ Research for Industry and the Wider Community - Food Science and Technology Excellence for a Sustainable \\ Bioeconomy
}

\begin{abstract}
In the traditional deductive approach in teaching any engineering topic, teachers would first expose students to the derivation of the equations that govern the behavior of a physical system and then demonstrate the use of equations through a limited number of textbook examples. This methodology, however, is rarely adequate to unmask the cause-effect and quantitative relationships between the system variables that the equations embody. Web-based simulation, which is the integration of simulation and internet technologies, has the potential to enhance the learning experience by offering an interactive and easily accessible platform for quick and effortless experimentation with physical phenomena. This paper presents the design and development of a web-based platform for teaching basic food engineering phenomena to food technology students. The platform contains a variety of modules ("virtual experiments") covering the topics of mass and energy balances, fluid mechanics and heat transfer. In this paper, the design and development of three modules for mass balances and heat transfer is presented. Each webpage representing an educational module has the following features: visualization of the studied phenomenon through graphs, charts or videos, computation through a mathematical model and experimentation. The student is allowed to edit key parameters of the phenomenon and observe the effect of these changes on the outputs. Experimentation can be done in a free or guided fashion with a set of prefabricated examples that students can run and self-test their knowledge by answering multiple-choice questions.
\end{abstract}

Keywords: Education; Food engineering; Web-based simulation

\section{Introduction}

Teaching in any engineering field almost always boils down to the process of deriving the mathematical equations that govern the phenomenon under study. Starting from simple laws and through the process of deduction, equations for progressively more complex phenomena are gen- erated. Students are tested on their understanding of this equation derivation process and their ability to pick the correct equations to solve any related problem.

Equations encompass the causal and quantitative relationships between the important variables in any phenomenon. However, knowing how the 
equations were derived or using the equations a few times to solve some textbook problems is not enough to unmask these relationships. Students may be able to correctly find the value of a variable $\mathrm{Y}$ by solving the correct equations using the known value of variable $\mathrm{X}$, but may fail to answer a question like: "what would happen to the value of $\mathrm{Y}$, if the value of $\mathrm{X}$ is decreased?" It is this kind of questions, however, that truly test the depth of knowledge and understanding of the studied phenomenon.

This understanding can be induced by observation, leaving the equations aside and 'experimenting' with the behavior of the system by manipulating its inputs and observing its outputs. Induction is the process of extracting global conclusions from local observations and, consequently, the ability to predict the behavior of a system at unfamiliar situations. Performing physical experiments is one way of inductive learning. However, there are many limitations on the number and complexity of experiments that can be performed in the lab and the degree of involvement of each student in the experiment. Experiments allow a close contact with the physical system, but they do not allow direct visualization of the variable dependencies, i.e. how the value of one variable is affected by a change in another. More importantly, physical experiments, especially in food engineering, usually manifest the interaction of multiple phenomena and it is difficult to isolate a single phenomenon for study. Simulation technology could be used as an alternative or could complement experimentation; simulation may have the disadvantage of being devoid of any physical contact with the actual system, but offers many advantages on the controllability of the experiment's content, the simplicity and speed at which these customized 'experiments' can be performed. A computer program embodying a simulated physical system hides the equations from the student; instead, it allows him or her to repetitively perform in a fast and inexpensive way multiple executions of the same experiment under variable conditions, to observe the outcome and, in the process, to discover and appreciate the causal relationships that equations embody. Virtual experimentation performed in the above way could effectively complement the traditional deductive approach in teaching physical phenomena in engineering. Web-based simulation is the integration of the Web with the field of simulation (Byrne, Heavey, \& Byrne, 2010). Its greatest advantage is accessibility: anyone with access to the internet can experiment with web-based simulations without having to install and learn to use software packages and without having to worry about licensing issues. Issues of security, stability, connection speed and GUI limitations (compared to standalone executables) may limit the usability of webbased simulation; however, the ease of access that it offers is by itself the single most important and adequate reason for considering it as a tool to deploy applications.

Using web-based simulation in science or engineering education is a recent but certainly not new idea. Kuljis and Paul (2001) list applications in education and training for distant students as one of the fields in which web-based simulation can be applied with cases that require huge quantities of data (e.g. meteorology) or collaborative projects from multiple sites being some other potential applications.

There is a multitude of methods and learning objectives that lie behind different applications of web-based simulations in education. In some instances, they are developed for visual appreciation only; virtual experiments are designed to provide a visual replication of a real (usually complex) phenomenon with not much educational content. In other cases, web-based simulation modules are developed to imitate exactly or to remotely control physical equipment or devices (usually electronic) found in a lab. Their purpose is to offer the student the exact same feeling as being in the laboratory (e.g. Benetazzo et al. (2000); Gillet, Ngoc, and Rekik (2005)). In other words, they serve as substitutes for the hands-on practical experience when working in the actual laboratory is not feasible.

We are using web-based simulation to enhance understanding of the theory, not as a substitute for experimentation. Visualization is used, not for seeing the physical system that you could otherwise see in the laboratory, but to 'see' the phenomenon behind the physical system and observe how that unfolds under varying conditions. The emphasis is in the behavior of the physical system as captured by its (hidden to the user) math- 
ematical model rather than the physical system itself.

There are many software tools that allow the development of model-based engineering simulations. There exist tools dedicated to specific applications and others which are generic. MAT$\mathrm{LAB}$ is a general-purpose mathematical package that allows the simulation of any engineering system and, thus, has numerous applications (Ibrahim, 2011). On the other hand, ANSYS Fluent is a specialized tool equipped with computational models for modeling fluid dynamics phenomena including those present in food processes (Sun, 2010). We are using both of these tools to develop our models.

This paper presents the first web-based educational modules developed as part of the FEPSIM (Food Engineering Process SImulation Modules) project. The modules represent very simple phenomena that could hardly be observed on their own in a physical system but which are of great importance for a student to understand. The modules are structured to provide an open, learner-centred environment where questions can be posed and answers can, hopefully, be obtained in the absence of a teacher.

\section{Materials and Methods}

A series of independent case studies (modules) has been (or, will be) developed as part of the FEPSIM project. Each module serves a different phenomenon of interest in food engineering. All modules are related to the areas of mass and energy balances, heat transfer and fluid mechanics. In each case, a mathematical model is developed and, along with it, a webpage that allows user interaction with the phenomenon through the model. All web pages supporting the different modules will have the necessary functionality to allow:

- visualization of the phenomenon,

- computation with the help of an underlying model,

- experimentation by the user.
Graphics, charts and/or videos are employed in each module and presented in the web page to help the user visualize the phenomenon under study. The mathematical model behind each module is developed to capture the dependencies between the important variables governing the phenomenon. The models are developed within MATLAB, ANSYS Fluent or by code. In some cases, the mathematical model is embedded into the web page and is run in real time. When this is not possible (e.g. with CFD models in Fluent), the models are run off-line for multiple combination of variable values and the webpage embeds the results of the simulation runs.

Figure 1 shows the introductory home page of the website at its current form. Image buttons and menu options navigate the user to the different modules. The website is developed to support the Greek and English languages and is available in the following web address: www.food.teithe. gr/fepsim (under development). This is an ongoing effort so the state of the website will be evolving in time. In the following subsections, the current state of three modules supporting mass balances and heat transfer in food processing are presented and analysed.

\subsection{Material balances}

\section{Educational Context}

Material and energy balances are everywhere in process and food engineering calculations; they formulate the basis for the development of any process model irrespectively of the model detail or resolution. This makes it imperative for students to master general balance equations before they can cope with the detailed models of specific operations.

Formulating and solving material and energy balance equations presents an impossible task to many students in process engineering. Empirical evidence suggests that this may be due to the fact that, unlike other topics in engineering, in mass and energy balances there are no predefined equations to use. Instead, the student must customize the general conservation equations not only to the specific problem but also to the available data for any specific problem. Even for the simplest cases, there are many dif- 


\section{FOOD ENGINEERING PROCESS SIMULATION MODULES}

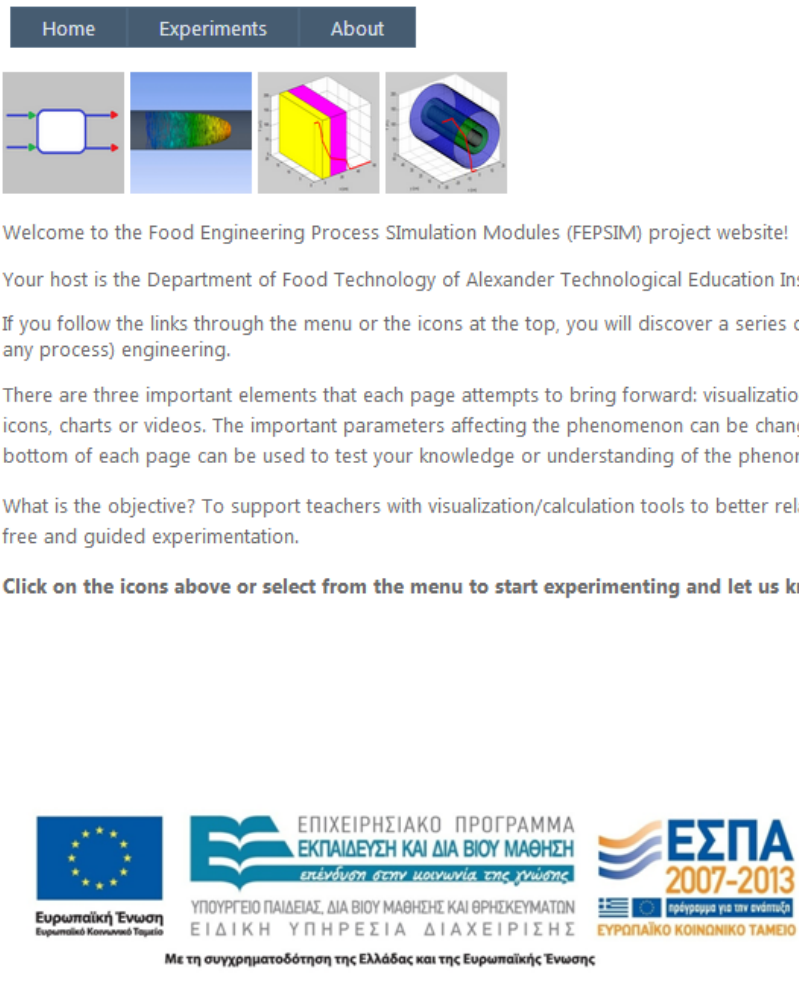

Figure 1: Introductory page of FEPSIM website

ferent ways in which a problem can be solved; only some of them, however, lead to fast solutions. That presents a challenge not only to the student but also to the teacher who must effectively guide and train the students in finding the fastest and most efficient solution strategy.

Consider a continuous process with an arbitrary number, $m$, of input and output streams carrying an arbitrary number, $n$, of components. Let us consider the case where no reactions take place into the process. The mass conservation principle suggests that the total input flow of each component must equal its total output flow. In other words, if $M_{j, i}$ is the mass flow rate of component $i$ in stream $j$, then the following equation holds:

$$
\sum_{j=1}^{m} M_{j, i}=0 \quad, \quad \forall i
$$

In the above equation, the flow rate is considered positive for input streams and negative for output streams. A similar equation can be written for the overall mass based on the stream flow rates $F_{j}$ :

$$
\sum_{j=1}^{m} F_{j}=0
$$

Using the total stream flow rates $F_{j}$ and the component mass fractions, $x_{j, i}$, equation (1) can be rewritten as:

$$
\sum_{j=1}^{m} x_{j, i} F_{j}=0 \quad, \quad \forall i
$$

In addition, component flow rates in each stream should obviously sum up to the total stream flow 
rate:

$$
\sum_{i=1}^{n} M_{j, i}=F_{j} \quad, \quad \forall j
$$

and, the component mass fractions in each stream should sum to 1 :

$$
\sum_{i=1}^{n} x_{j, i}=1 \quad, \quad \forall j
$$

All above equations are candidate equations to be used to solve a mass balance problem around a single process but, obviously, they are not independent so, they cannot be used simultaneously. For every problem and depending on the available data, only a subset of the above equations can be used to yield the values of all variables. When solving the problem by hand, however, it is not always easy to spot which of the above equations can preferentially be employed to find the solution in the most efficient way.

A teacher could employ heuristic rules to guide the students; for example, from all components in the process, the best candidates for balance equations with the smallest number of unknowns are the components for which the most data on their mass fractions or flowrates is available. Still, this knowledge can come only from laborious supervised practice but how often can you have a teacher guiding you in every problem?

\section{Web-based simulation module}

The objective of the mass balance module is to offer virtual guidance to the student in solving balances around a simple process. Figure 2 shows the webpage interface of this module. The user of this module would have to first select the input/output structure of the process by clicking one of the five picture buttons at the top and, then specify, the components present in the process. The graphical display of the process shows the selected structure and, for each stream, a total flow rate field and a table with each component's mass fraction and rate.

To use the page, the user will have to start entering whatever data are available for the problem at hand. As this process goes on, an embedded algorithm (developed by code in $\mathrm{C \#}$ ) is run to identify what other variables can be calculated using the provided data with the help of any of the above equations. A color-coding scheme is employed to differentiate variables set by the user (red font), variables calculated (green font) and variables that could potentially be calculated (orange background). The idea behind this design is that, whenever a field in orange background shows up, the user should wonder how it is possible to have this value calculated. By pressing the Calculate Next button the answer can be revealed both in terms of the value (the orange background gives way to a 'green' value) and in terms of what equation(s) can be used to calculate this value; the equations will show up in a flying tooltip if the user moves the mouse over the variable field.

A series of predefined problems posed as multiple-choice questions can be used by the student for practice. In addition, a student can use the webpage to solve their own mass balance problem for a single process with no reactions and at most 4 input or output streams. The webpage is designed to provide structure and discipline to the process of solving a mass-balance problem, virtues, which, after adequate experimentation could be learned and transferred into solving more complicated problems by hand.

\subsection{Heat Transfer}

\section{Educational Context}

Heat transfer is present in almost all food processes and, therefore, is an important topic in food engineering. Food technologists should master the mechanisms underlying heat transfer and comprehend the relative significance of all factors affecting its distribution and rate. In any typical food process, modeling the heat transfer mechanisms through the energy balance is a quite complex process involving the construction and solution of 3-D partial differential equations. As usually, teaching of a complex subject involves its decomposition into simpler, idealized sub-entities that can be modeled and studied independently. So, for example, students in heat transfer are first exposed to the basic mechanisms (conduction, convection, radiation) in isolation from multi-layer) solid surface separating two fluid phases. As defined in any basic heat transfer text book (e.g. Pitts and Sissom, 1998), 
$6 \mid$ Koulouris et al.

SINGLE-PROCESS MASS BALANCES
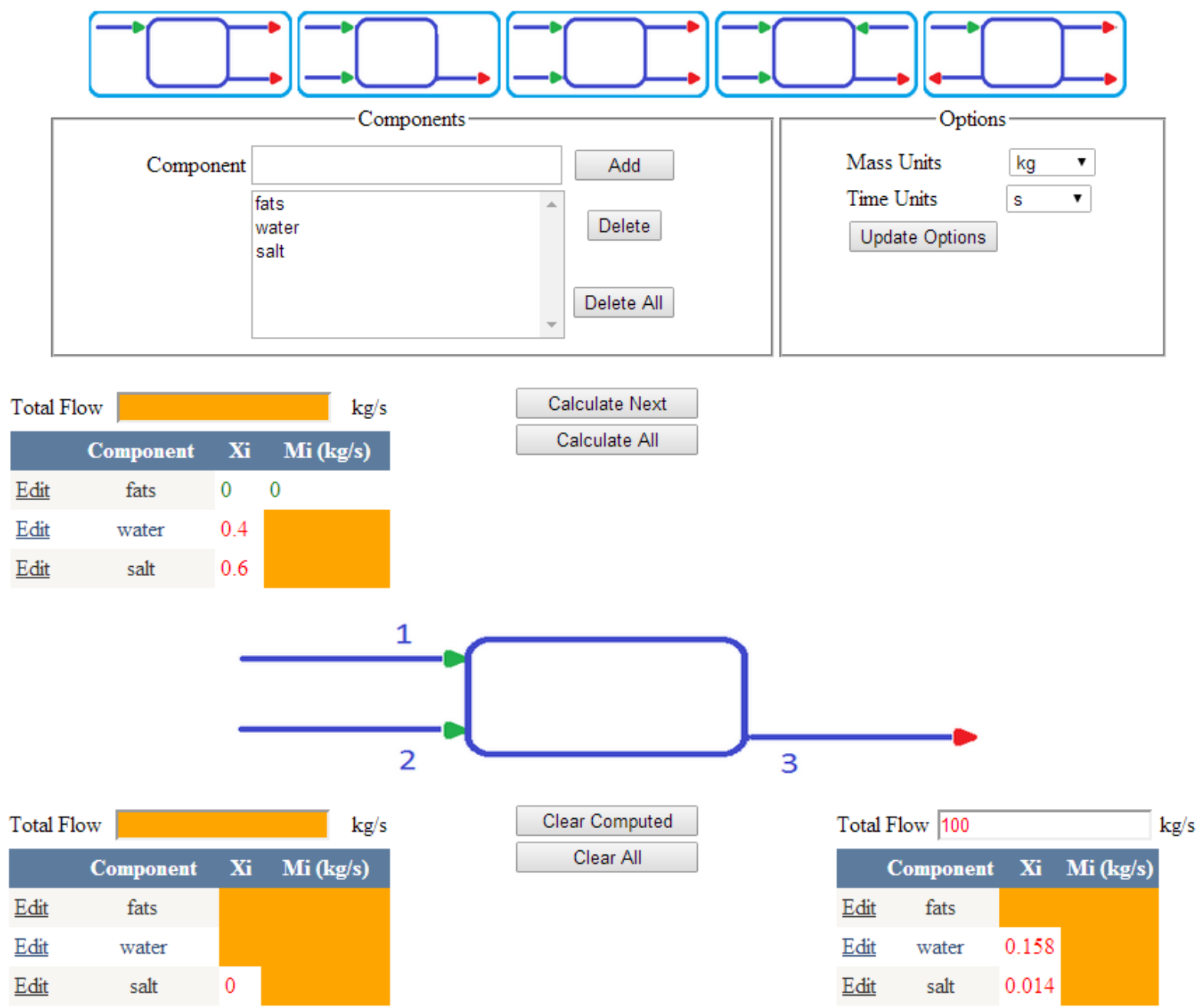

Figure 2: Webpage interface for single process mass balance module 
the heat transfer rate, $q$, is driven by the temperature difference, $\Delta \mathrm{T}$, between the two fluids, and is proportional to the heat transfer area, $A$, and the overall heat transfer coefficient $U$ :

$$
q=U A \Delta T
$$

The overall heat transfer coefficient embeds all the resistances in heat transfer including the conductive solid layers characterized by the layer's thermal conductivity, $k$, and the fluid phases characterized by the convective heat transfer coefficient, $h$. The form of the overall heat transfer coefficient defining equation depends on the geometry. For planar layers:

$$
U=\frac{1}{\frac{1}{h_{i}}+\sum_{i} \frac{L_{i}}{k_{i}}+\frac{1}{h_{o}}}
$$

where, $L_{i}$ is the thickness of the convective layer $i$. In cylindrical configurations, $U$ is given by:

$$
U=\frac{1}{\frac{r_{n}}{r_{i} h_{i}}+r_{n} \sum_{j=2}^{n} \frac{1}{k_{j}} \ln \left(\frac{r_{j}}{r_{j-1}}\right)+\frac{1}{h_{o}}}
$$

where, $r_{i}$ is the radius of layer $i$. Equation (8) assumes that the heat transfer area is calculated at the outer surface with radius $r_{n}$.

Students are not only expected to be able to use the above relations to compute the overall heat transfer coefficient and the heat flux but also understand the relative effect of the parameters involved and, more importantly, how the temperature profile on the walls is affected by changes in these parameter values. It is a typical misconception among the students that an insulating material (with a small value of heat conductivity, $k$ ) would have a smaller temperature difference between its two surface walls than other noninsulating layers. This misconception arises from the fact that students learn to relate heat transfer rates with temperature differences $(\Delta T)$, so, an insulating material must cause a small heat rate and, therefore, small $\Delta \mathrm{T}$. They do not realize, however, that in a given steady-state case, the heat transferred is the same everywhere and that the presence of the insulating material causes a relatively large temperature difference between its two sides while a heat conducting material maintains an almost constant temperature profile across its width.

In cylindrical coordinates, there are two competing factors that determine the value of heat rate as the thickness of the insulating layer increases. The increase in thickness of a layer may provide extra insulation but it also causes an increase in the heat transfer area which, in turn, means larger heat transfer rate. Under certain circumstances, the surface increase dominates and it is possible to have greater heat losses even when extending the insulating layer. We developed two simulation modules to reveal to the student the real dependencies and help the student get rid of such misconceptions.

\section{Web-based simulation module}

MATLAB was used to develop the corresponding mathematical models and charting functionality to support the visualization of the steady-state heat transfer process in planar and cylindrical geometries. The scope of the module was to expose the phenomenon to the users and allow them to experiment freely with it without requiring any coding or knowledge, on their part, of MATLAB. The MATLAB Builder NE toolbox was used to incorporate the MATLAB model and graphics into an ASP.NET webpage.

Figures 3 and 4 present the web page interfaces for steady-state convective/conductive heat transfer for planar and cylindrical configurations respectively. A description of the interface elements in both cases follows. The graph at the bottom of the window shows the physical wall(s) through which heat is transferred with the temperature profile projected on the front surface. Edit controls at the top of the page allow the user to change the internal and external fluid temperatures and the values of the convective heat transfer coefficients on both sides. These values are expected to change infrequently. What the user is expected mainly to experiment with are the number of layers (up to three) and the thickness and heat conductivity of each layer all of which can be edited through the corresponding controls in the window. With every change in any value and after the user clicks the Refresh button, the model recalculates the overall heat transfer coefficient and the main graph showing 
FOOD ENGINEERING PROCESS SIMULATION MODULES

\section{Home Experiments About}

\begin{tabular}{|c|c|c|c|}
\hline $\mathrm{T}_{\mathrm{i}}\left({ }^{\circ} \mathrm{C}\right)$ & 200 & $\mathrm{~T}_{0}\left({ }^{\circ} \mathrm{C}\right)$ & 25 \\
\hline$h_{i}\left(W / m^{2 \circ} \mathrm{C}\right)$ & 10 & $\mathrm{~h}_{\mathrm{o}}\left(\mathrm{W} / \mathrm{m}^{2 \circ} \mathrm{C}\right)$ & 20 \\
\hline Number of Layers & 2 V & Refresh & Clear \\
\hline $\mathrm{L}_{1}(\mathrm{~cm})$ & 5 & $\mathrm{k}_{1}\left(\mathrm{~W} / \mathrm{m}^{\circ} \mathrm{C}\right)$ & 0.1 \\
\hline $\mathrm{L}_{2}(\mathrm{~cm})$ & 20 & $\mathrm{k}_{2}\left(\mathrm{~W} / \mathrm{m}^{\circ} \mathrm{C}\right)$ & 2 \\
\hline $\mathrm{U}\left(\mathrm{W} / \mathrm{m}^{2 \circ} \mathrm{C}\right)$ & 1.33 & $\mathrm{q} / \mathrm{A}\left(\mathrm{W} / \mathrm{m}^{2}\right)$ & 233.33 \\
\hline
\end{tabular}
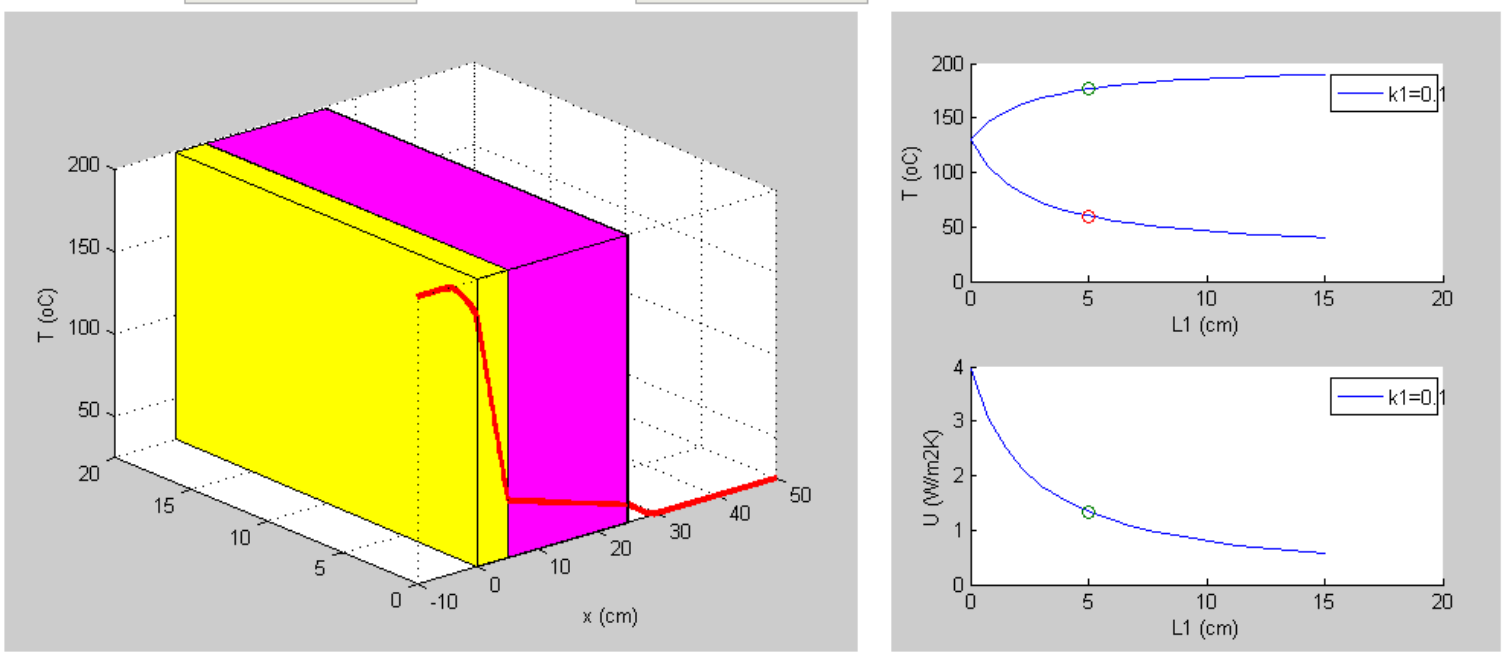

Figure 3: Webpage interface for the steady-state heat transfer in planar geometry module

the walls and the temperature profile is updated. At the right hand side of the main chart, there exist two additional charts which show how temperatures at the inner and outer surface of the wall as well as the overall heat transfer coefficient change with the wall thickness and the heat conductivity of the corresponding layer. Since there may be more than one layer in the model, the page detects which layer the user modifies and displays the charts with respect to that layer. So, for example, if the user keeps changing the values of thickness and/or conductivity of the second layer, then the charts will show how temperatures and $U$ change with respect to the second layer. The charts show continuous curves for a range of wall thickness values and for different conductivity values; the specific values tested by the user are shown as dots on these curves. In this way, the user can easily recognize the effects of changing the wall features on heat transfer. For example, as shown in Figure 3, the experiment makes apparent to the student that as the wall thickness increases, the value of the overall heat transfer coefficient decreases (as expected) but also the temperature difference between the two walls of the solid layer increases. As the wall becomes thinner or the solid's heat conductivity is increased, the burden of maintaining a given temperature gradient between the two fluids separated by the solid surface moves to the convective terms in the fluids side. In Figure 4, the competing effects of increasing the wall thickness 
Web-based food engineering education modules $\mid 9$

\section{FOOD ENGINEERING PROCESS SIMULATION MODULES}

\begin{tabular}{|c|c|c|c|}
\hline Experiments & About & & \\
\hline $\mathrm{T}_{\mathrm{i}}\left({ }^{\circ} \mathrm{C}\right) 200$ & $\mathrm{~T}_{\mathrm{o}}\left({ }^{\circ} \mathrm{C}\right) 25$ & & \\
\hline$h_{i}\left(W / m^{2 \circ} \mathrm{C}\right) 1000000$ & $\mathrm{~h}_{\mathrm{o}}\left(\mathrm{W} / \mathrm{m}^{2 \circ} \mathrm{C}\right) 4$ & & \\
\hline $\mathrm{r}_{\mathrm{i}}(\mathrm{cm}) 1$ & & & \\
\hline Number of Layers 2 & & & \\
\hline$\Delta \mathrm{r}_{1}(\mathrm{~cm}) 0.1$ & $\mathrm{k}_{1}\left(\mathrm{~W} / \mathrm{m}^{\circ} \mathrm{C}\right) 1000$ & Refresh & Clear \\
\hline$\Delta \mathrm{r}_{2}(\mathrm{~cm}) 2$ & $\mathrm{k}_{2}\left(\mathrm{~W} / \mathrm{m}^{\circ} \mathrm{C}\right) 0.072$ & & \\
\hline $\mathrm{U}\left(\mathrm{W} / \mathrm{m}^{2 \circ} \mathrm{C}\right) 1.44$ & $\mathrm{q} / \mathrm{L}(\mathrm{W} / \mathrm{m}) 48.97$ & & \\
\hline
\end{tabular}
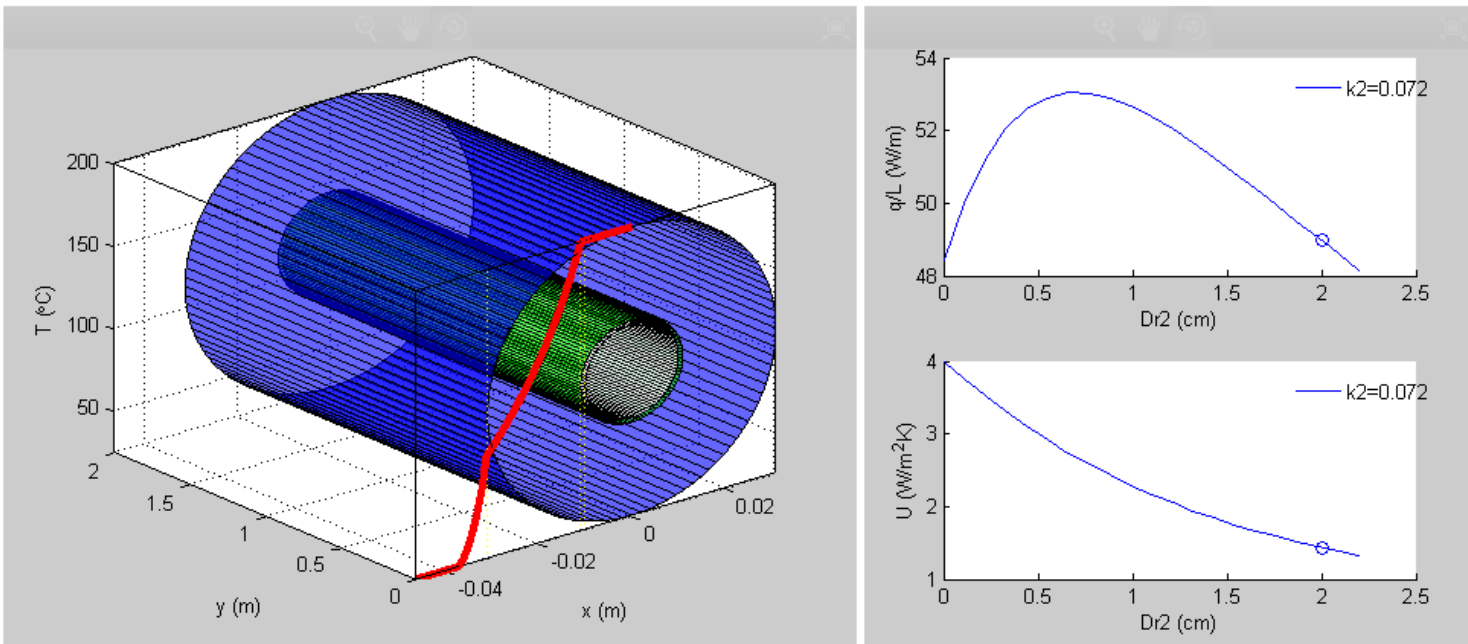

Help Instructions

Figure 4: Webpage interface for the steady-state heat transfer in cylindrical geometry module

and the inevitable increase in the surface area become apparent. Although the value of the overall heat transfer coefficient decreases monotonically as wall thickness increases, the profile of heat rate per unit length shows a maximum demonstrating the fact that increased heat losses are possible even when adding more insulating material around a pipe.

\section{Results and Discussion}

The three case studies presented in this paper constitute the first in a series of ten educational web-based modules that will be developed as part of the FEPSIM project. The scientific content of all modules will evolve around basic phenomena in food engineering such as heat transfer and flow mechanics. In all cases, the web-based module is designed to allow the user to experiment with the physical system (and its underlying model) by manipulating the values of input variables and observe the effect on the evolution of the phenomenon through the visual components of the webpage. This interactive experimentation can be done in two ways. In free interactive mode, users are completely free to 'play' with the model by changing any parameter values they 


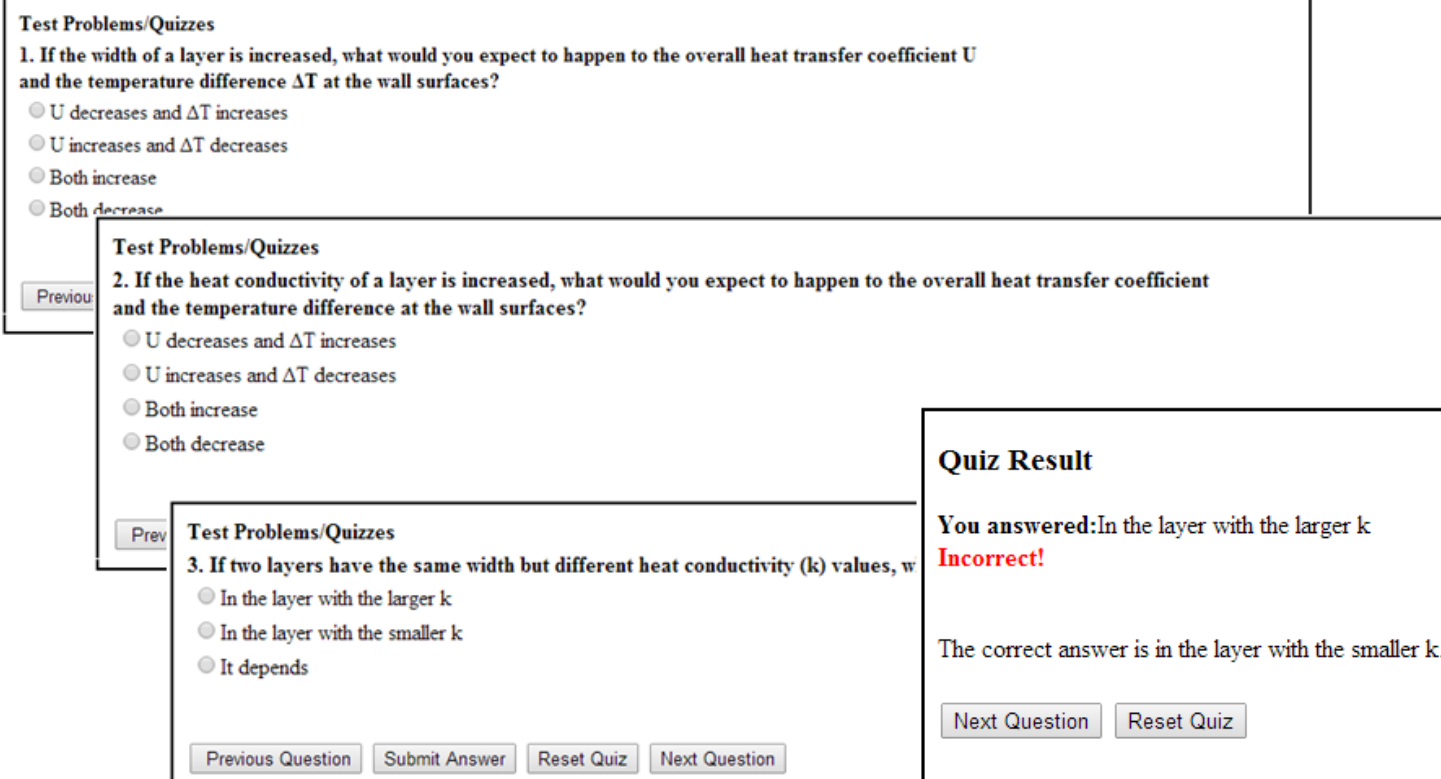

Quiz Result

You answered:In the layer with the larger $\mathrm{k}$

Incorrect!

The correct answer is in the layer with the smaller $k$

Next Question Reset Quiz

Figure 5: Webpage quizzes on the heat transfer module

want and observing the effect on the output variables. Even if changes are random, it is still possible for some noteworthy behavior to occur that will prompt the user to search for an explanation. Alternatively, users could use a set of pre-defined quiz questions or problems available at the bottom of the webpage like the ones shown in Figure 5; these quizzes and problems are formed as multiple-choice questions and are developed to guide users to observe important effects that offer insight into the phenomenon under study. In addition, each module provides links to a short theoretical analysis of the phenomenon and to instructions on how to use the webpage.

It is hoped that with the help of these modules, teaching of these topics can be enhanced with visual and real-time exposition of the evolution of the relevant phenomena. Students will have at their disposal an environment where they can learn by themselves and test their knowledge. The extent to which this will be achieved will be apparent after the completion of the modules and the exposition of students to this material though a formal classroom setting.

\section{Conclusions}

As claimed by Leung (2003), the use of simulation in education serves the purpose of shifting the learning paradigm from direct instruction to a more open-ended and learner-centred process to permit sustained exploration by the learners. This is in line with what we are also trying to achieve with the development of modules such as the ones presented in this paper: allow interactive experimentation with a phenomenon, offer a personalized experience to the student, and provide feedback in many different forms so that students can learn on their own. The use of these modules is not supposed to substitute the analysis into the classroom. Instead, it is intended to complement and strengthen the theoretical exposure with the inductive knowledge that comes from practicing freely with the phenomenon at hand.

\section{Acknowledgements}

This research has been co-financed by the European Union (European Social Fund - ESF) 
Web-based food engineering education modules $\mid 11$

and Greek national funds through the Operational Program "Education and Lifelong Learning" of the National Strategic Reference Framework (NSRF) - Research Funding Program: ARCHIMEDES III Investing in knowledge society through the European Social Fund.

\section{References}

Benetazzo, L., Bertocco, M., Ferraris, F., Ferrero, A., Offelli, C., Parvis, M., \& Piuri, V. (2000). A web-based distributed virtual educational laboratory. IEEE Transactions on Instrumentation and Measurement, 49(2), 349-356. doi:10.1109/19. 843077

Byrne, J., Heavey, C., \& Byrne, P. J. (2010). A review of web-based simulation and supporting tools. Simulation Modelling Practice and Theory, 18(3), 253-276. doi:10. 1016/j.simpat.2009.09.013

Gillet, D., Ngoc, A. V. N., \& Rekik, Y. (2005). Collaborative web-based experimentation in flexible engineering education. IEEE Transactions on Education, 48(4), 696704. doi:10.1109/.TE.2005.852592

Ibrahim, D. (2011). Engineering simulation with matlab: improving teaching and learning effectiveness. Procedia Computer Science, 3, 853-858. World Conference on Information Technology. doi:10.1016/j.procs. 2010 . 12.140

Kuljis, J. \& Paul, R. J. (2001). An appraisal of web-based simulation: whither we wander? Simulation Practice and Theory, 9(12), 37-54. doi:10 . 1016 / S0928 - 4869(01) 00032-5

Leung, A. C. K. (2003). Contextual issues in the construction of computer-based learning programs. Journal of Computer Assisted Learning, 19(4), 501-516. doi:10. 1046/j.0266-4909.2003.00053.x

Pitts, D. R. \& Sissom, L. E. (1998). Schaum's outline of heat transfer. McGraw-Hill.

Sun, D. W. (2010). Computational fluid dynamics in food processes. CRC Press. 